Crise et catastrophe dans l'argumentation publicitaire

Krise und Katastrophe in der Werbeargumentation

Crises and Disasters in Advertising Argument

\title{
Nathalie Schnitzer
}

\section{(2) OpenEdition}

\section{Journals}

Édition électronique

URL : http://journals.openedition.org/ceg/2446

DOI : $10.4000 /$ ceg. 2446

ISSN : 2605-8359

Éditeur

Presses Universitaires de Provence

Édition imprimée

Date de publication : 26 octobre 2017

Pagination : 221-234

ISBN : 979-10-320-0129-5

ISSN : 0751-4239

\section{Référence électronique}

Nathalie Schnitzer, « Crise et catastrophe dans l'argumentation publicitaire », Cahiers d'Études

Germaniques [En ligne], 73 | 2017, mis en ligne le 26 avril 2019, consulté le 21 novembre 2020. URL :

http://journals.openedition.org/ceg/2446 ; DOI : https://doi.org/10.4000/ceg.2446 


\section{Crise et catastrophe dans l'argumentation publicitaire}

Nathalie SCHNITZER

Aix Marseille Univ, ECHANGES, Aix-en-Provence, France

\section{Introduction}

Rien ne semble à première vue plus éloigné de la notion de crise que l'univers euphorisant de la publicité, où tout est fait pour mettre le consommateur dans des dispositions favorables à l'achat du produit. Mais lorsqu'on y regarde de plus près, on s'aperçoit que nombre de campagnes ne se limitent pas à l'évocation d'un monde radieux et que les situations de crise, voire les catastrophes, constituent également un ressort de l'argumentation publicitaire. L'objectif du présent article est donc d'élaborer une typologie des campagnes publicitaires qui ont une affinité avec la notion de crise ou de catastrophe, en s'appuyant sur un corpus composé d'affiches et de spots publicitaires principalement français et allemands.

\section{Publicité et crise}

Superficielle ou profonde, passagère ou durable, individuelle ou collective, économique ou politique, de société ou de civilisation, la crise est une notion difficile à cerner tant ses causes et ses formes sont multiples. Pour mieux en percevoir les contours, on s'appuiera ici sur un texte d'Edgar Morin intitulé « Pour une crisologie » et publié en 1976 dans un numéro de la revue Communications consacré précisément à la notion de crise. Dans ce bref essai, réédité ailleurs depuis ${ }^{1}$, il présente la crise comme une perturbation du système, lui-même défini comme "un ensemble organisé par l'interrelation de ses constituants » (Morin 1976: 150). L'équilibre du système tient non seulement à la présence de forces complémentaires, mais aussi à celle de forces antagonistes «maintenues et organisées » (Morin 1976: 150), qui représentent une menace potentielle pour l'existence du système. La crise surgit lorsque les forces antagonistes prennent le dessus sur les forces complémentaires. Ce déséquilibre, s'il perdure et s'aggrave, est

1. Cet article d'Edgar Morin a été réédité récemment (Morin et L’Yvonnet 2016). 
susceptible d'entraîner la mort du système: " On ne peut concevoir d'organisation sans antagonisme, mais cet antagonisme porte en lui, potentiellement, et tôt ou tard inévitablement, la ruine et la désintégration du système » (Morin 1976: 152). La crise est donc susceptible de se muer en catastrophe. Comme le souligne René Thom dans le même numéro de Communications, crise et catastrophe se distinguent fondamentalement tout en entretenant des liens étroits : la première peut certes demeurer sournoise et latente tandis la seconde est toujours un fait patent, mais cela n'empêche pas la crise d'être "souvent l'annonciatrice de la catastrophe, qu'elle précède, ou qu'elle provoque » (Thom 1976:34).

On pourrait penser que les publicitaires, dont l'objectif premier est de faire l'éloge du produit afin de susciter l'acte d'achat, n'ont aucun intérêt à évoquer des situations de crise et donc à attirer l'attention du consommateur sur des déséquilibres constituant un danger mortel pour le système. II paraît a priori plus habile de le mettre en confiance pour le placer dans des dispositions favorables vis-à-vis de la marque. II n'a pourtant pas été difficile, pour les besoins de cette étude, de rassembler un corpus d'une cinquantaine d'exemples en lien avec le sujet (dont une partie seulement pourra être analysée ici), ce qui s'explique sans doute en partie par le jeu des vases communicants, la publicité ne pouvant pas rester totalement étanche à l'omniprésence actuelle de la crise dans les médias, mais pas seulement. Les exemples collectés, qui couvrent une période de plus d'un demi-siècle (des années 60 à nos jours), montrent en effet que crise et publicité entretiennent depuis longtemps des liens complexes que cet article tente de démêler.

\section{Publicité et argumentation}

Les enchaînements argumentatifs qui sous-tendent le discours publicitaire peuvent généralement être représentés à l'aide du schéma élémentaire suivant, dérivé du modèle de Toulmin (1993)²:

$\begin{array}{cccc}\text { Argument }[\text { donnée] } \rightarrow & \text { Donc } & \text { (probablement) } \rightarrow & \begin{array}{c}\text { Conclusion } \\ \text { Le produit } X \text { possède } \\ \text { telle propriété } \\ \text { remarquable }\end{array} \\ \text { Le produit X }\end{array}$

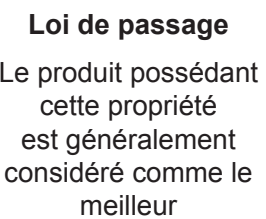

\section{(Restriction)}

à moins qu'il ne possède tel défaut qui supplante ses qualités

2. Voir aussi la présentation de ce modèle par Adam et Bonhomme dans leur ouvrage consacré à l'argumentation publicitaire (1997: 111-112). 
Ce schéma appelle deux remarques. Premièrement, l'argumentation dont il est question ici est davantage persuasive que convaincante (cf. Perelman et Olbrechts-Tyteca 1992: 34-40), puisqu'elle est conçue pour un auditoire particulier (la cible publicitaire) et orientée vers l'action (l'acte d'achat). Deuxièmement, il faut avoir à l'esprit que l'enchaînement argumentatif n'est généralement pas immédiatement détectable pour le destinataire non averti, car dans la pratique les annonceurs empruntent volontiers des chemins détournés pour persuader les destinataires de l'excellence de leur produit et de sa supériorité vis-à-vis de la concurrence. Tantôt les arguments ne sont pas explicitement énoncés, tantôt c'est la conclusion qui demeure implicite, quant à la «loi de passage » reliant l'argument à la conclusion, elle ne fait pas toujours l'objet d'une énonciation. Le lien entre ce que l'on nomme couramment l'argument de vente et un enchaînement argumentatif véritable se révèle parfois ténu ${ }^{3}$.

Pour comprendre comment ces publicités fonctionnent sur le plan argumentatif, il faut alors se placer dans la perspective de leur réception, en particulier pour les publicités qui n'accordent que très peu de place aux contenus verbaux, obligeant le spectateur à activer ses compétences intersémiotiques. C'est en effet au destinataire qu'est confiée la tâche de reconstruire l'enchaînement argumentatif à partir des divers fragments (indices graphiques, sonores, visuels) mis à sa disposition. Dans un article au titre volontairement provocateur «Ist ein "Muh!" ein relevantes Argument? », Bo Andersson fournit un bon exemple de ce travail de reconstruction de l'argumentation à partir d'un spot publicitaire pour une marque suédoise de produits laitiers (Andersson 1997). Cette campagne présente aussi l'avantage de mettre en scène un monde parfait, donc très éloigné de toute idée de crise.

\section{Le degré zéro de la crise en publicité}

Pour Bo Andersson, le genre publicitaire est argumentatif par essence. Au-delà de la fonction informative, faire connaître la marque ou le produit, l'objectif poursuivi est en effet fondamentalement d'agir sur le destinataire en l'amenant à porter un jugement positif sur la marque et / ou sur un produit de la marque. Les moyens mis en œuvre ne sont pas seulement textuels. L'image publicitaire joue également un rôle important, parfois même prédominant. Dans le cas d'un spot publicitaire, le dispositif sémiotique mis en place pour emporter l'adhésion du destinataire intègre l'environnement sonore, musique d'ambiance, bruits de fond divers, voire cris d'animaux - comme ici :

Bregott: changement d'équipe (1997) ${ }^{4}$

3. Voir à ce propos les réserves émises par Desîlets et Gauthier (2013) sur le caractère argumentatif de certaines publicités.

4. Film publicitaire: [https://youtu.be/KYQKA4gONME], dernière consultation le 30.10.2016. Tous les liens cités dans cet article ont été consultés à la même date. 
Dans le document analysé par Bo Andersson, on découvre une colline verdoyante au sommet de laquelle se croisent deux petits troupeaux de vaches, les unes s'éloignant, les autres s'approchant du spectateur en meuglant. Les seuls éléments verbaux contenus dans cette publicité apparaissent à l'image: "Skyftbite ¡ Bregottfabriben » (changement d'équipe dans les fabriques Bregott) en milieu de spot, puis «Bara naturliga råvaror » (ingrédients $100 \%$ naturels) en fin de spot ${ }^{5}$. Bo Andersson considère qu'un argument est pertinent à la condition suivante:

die Relevanz des Arguments hängt [...] damit zusammen, ob sich eine akzeptable Schlussregel für die Verknüpfung von Argument und Konklusion finden lässt » (Andersson 1997: 21).

En s'appuyant sur cette définition, l'auteur répond positivement à la question posée par le titre de son article: le meuglement des vaches (dans le décor champêtre approprié) constitue bel et bien un argument en faveur de la conclusion "les produits Bregott sont naturels», en vertu de la loi de passage "les animaux élevés dans la nature fabriquent des produits naturels »; les étapes suivantes de la macro-argumentation que le destinataire est incité à reconstruire peuvent se résumer ainsi: ce qui est naturel est bon, ce qui est bon mérite d'être acheté, achetez Bregott! Certes, assimiler le meuglement d'une vache à un argument peut paraître abusif, mais on retiendra l'idée que le «meuh! » joue effectivement le rôle de déclencheur du processus argumentatif qu'il revient au destinataire de verbaliser. On pourrait parler ici d'un argument en puissance. On remarquera d'ailleurs que dans des campagnes plus récentes, le meuglement reste de rigueur et fonctionne comme une signature sonore de la marque Bregott ${ }^{6}$.

La publicité Bregott et ses vaches qui gambadent en liberté dans des prairies verdoyantes, se relayant pour produire naturellement du lait, est prototypique de nombreuses publicités mettant en scène un monde merveilleux où des produits présentés comme étant du terroir sont fabriqués dans des fermes pittoresques par des paysans heureux, parfois assistés de leurs animaux. Le public français se souvient sans doute de la publicité Milka dans laquelle le randonneur aperçoit une marmotte en train d'emballer une tablette de chocolat dans du papier d'aluminium:

Milka: la marmotte $(1998)^{7}$

Le spectateur n'est évidemment pas dupe, mais accepte volontiers de se laisser divertir par cette vision de la fabrication du chocolat digne d'un conte de fées. Pour désigner ces publicités qui se veulent avant tout divertissantes, Jacques Séguéla a forgé le terme advertainment (Séguéla 2004 [en ligne]).

Sans aller aussi loin que la publicité Milka dans la construction d'un univers purement fictionnel, de nombreuses campagnes embellissent volontiers la réalité

5. Pour la description détaillée du spot, voir Andersson (1997: 25).

6. Voir notamment:

Bregott (2013) Hashtag i Bregottfabriken. [https://youtu.be/OfFNnn8kock].

Bregott (2014) Opinionssvängning i Bregottfabriken. [https://youtu.be/6nDsX7kkKzM].

7. Film publicitaire: [https://youtu.be/_Qg3Rk-B09o]. 
pour faire rêver le consommateur. Si l'évocation d'un monde parfait crée des conditions propices à l'achat du produit, elle se heurte également à un paradoxe: comment persuader la cible publicitaire que l'absence de tout problème doit tout de même déboucher sur l'achat d'un produit? Faut-il vraiment que l'herbe soit encore plus verte, le linge encore plus blanc, le mari et les enfants encore plus heureux, bref la vie encore plus belle qu'elle ne l'est déjà? Aussi divertissante soient-elles, ces images d'Épinal risquent à la longue de lasser le spectateur.

\section{La crise sans gravité: un monde presque parfait}

Sans pour autant renoncer au caractère léger et divertissant de la publicité, il s'agit de persuader le consommateur de l'existence d'un problème que le produit est censé résoudre. Un moyen d'y parvenir est de présenter ce problème comme étant de faible gravité, donc peu anxiogène. Dans une campagne diffusée à la télévision française dans les années 80 , les personnages se plaignent d'un manque de tonus en chantant "J'sais pas c'qu'il y a, j'suis raplapla... » pour promouvoir l'eau de Vittel:

Vittel: buvez, éliminez (1984) ${ }^{8}$

La mise en scène façon comédie musicale (sur des paroles de Gotainer) est fidèle à l'esprit de l'advertainment, même si le problème est explicitement nommé. II y a plus grave dans la vie que d'être "tout mou ", "raplapla » ou "flagada », cependant ce petit désagrément ne demande qu'à être surmonté grâce à la pratique d'une activité sportive et à l'absorption régulière de l'eau de Vittel, qui aide à "éliminer», d'où l'injonction au consommateur: "Buvez, éliminez! ». Le schéma argumentatif est simple: vous êtes raplapla (argument), donc buvez Vittel (conclusion), puisque Vittel vous aide à retrouver la vitalité qui est en vous (loi de passage).

Cette stratégie argumentative consiste donc à mettre l'accent sur la capacité du produit à résoudre un problème auquel le consommateur est confronté. Cette technique de la «Problem-Lösung »(Gau 2007: 75) est très utilisée dans les publicités pour les produits ménagers (lessives et autres détergents). On pense à la scène du mari ou des enfants arborant des vêtements couverts de taches, un spectacle qui devrait accabler la brave mère de famille si la lessive $X$ ou $Y$ ne promettait pas de les faire disparaître à coup sûr:

Vanish: Lippenstift (2009) ${ }^{9}$

Mais la volonté de marquer davantage les esprits et de favoriser la mémorisation incite les publicitaires à forcer le trait; le petit désagrément prend alors de l'ampleur jusqu'à se transformer en véritable crise rendant le produit indispensable: aux grands maux, les grands remèdes! Autrement dit, le procédé consiste à fabriquer

8. Film publicitaire: [https://youtu.be/_cLe_y268M4].

9. Film publicitaire: [https://youtu.be/-0_PpQE4sKY]. 
une crise pour mieux la résoudre. Rien de plus simple si l'on considère que la crise n'existe pas comme telle, mais qu'elle relève d'une perception subjective, ou pour reprendre la définition de René Thom (qui souligne que le phénomène ne touche pas les objets, mais les sujets): "Est en crise tout sujet dont l'état [...] est perçu par le sujet lui-même comme une menace à sa propre existence » (Thom 1976: 35). Si la crise est d'abord une affaire de perception, l'enjeu pour les publicitaires est donc de rendre la crise perceptible par le public cible.

\section{La construction de la crise en publicité}

La stratégie ne consiste plus, comme précédemment, ni à nier ni à minimiser le problème, mais au contraire à l'amplifier en lui donnant la forme d'une crise plus ou moins aigüe, voire d'une catastrophe. II convient ici de rappeler ce qui distingue radicalement crise et catastrophe: la catastrophe est un «fait » patent qui atteint la "structure » du système, alors que la crise, qui touche la « fonction » du système, peut quant à elle rester "latente ou sournoise » (Thom 1976: 34). Le propre de la catastrophe, outre son caractère soudain et brutal, est qu'elle provoque des bouleversements empêchant un retour à l'état antérieur, tandis que la crise est un processus évolutif susceptible, selon les circonstances et la manière dont elle est gérée, de s'aggraver ou de se résorber en laissant ou non des séquelles. Par ailleurs, si certaines catastrophes sont précédées de signes avant-coureurs, d'autres sont totalement imprévisibles et n'auraient pas pu être évitées grâce à une meilleure gestion de la crise. Autrement dit, lorsque le sujet traverse une crise, il peut encore prendre la bonne ou la mauvaise décision, lorsqu'une catastrophe s'abat sur lui, il est totalement démuni, il ne peut que la subir. Le caractère imprévisible de certaines catastrophes en fait des événements particulièrement anxiogènes et on peut d'autant plus s'étonner de leur utilisation en publicité. Certains spots publicitaires s'appuient pourtant sur de véritables scénarios catastrophes, comme on va le voir dans la suite. On distinguera deux principaux cas de figure: soit la crise préexiste et rend nécessaire l'utilisation du produit, soit c'est la non-utilisation du produit qui provoque la crise.

\section{La crise appelle le produit (crise $>$ produit)}

Le consommateur est confronté à une crise et le produit vient à point nommé pour, selon les cas, résoudre la crise, éviter la catastrophe ou aider le clientvictime à se reconstruire. Ce schéma est un classique en publicité, comme l'illustre cette campagne culte pour les cigarettes de la marque HB, qui emprunte les codes du comique burlesque:

HB: im Laden (années $60 / 70)^{10}$

10. Film publicitaire: [https://youtu.be/VKo4s_7-Afc]. 
Il s'agit d'une série de dessins animés dans lesquels le personnage de Bruno est invariablement confronté à des situations exaspérantes: il se cogne, veut rattraper un objet, perd l'équilibre, tombe à la renverse, etc. Plus il tente de maîtriser la situation, plus elle lui échappe. II gesticule, il peste dans une langue incompréhensible, et sort finalement de ses gonds (" er geht an die Decke » au sens propre et au figuré). C'est alors que surgit le HB-König, sorte d'ange gardien à la tête couronnée, qui le ramène à la raison en s'écriant: "Halt, mein Freund! Wer wird denn gleich an die Decke gehen? Greife lieber zur HB! Dann geht alles wie von selbst. » Et en disant ces mots, il tend à Bruno un paquet de cigarettes de la marque en question. Bruno allume une cigarette et retrouve immédiatement ses esprits. Et la voix off de poursuivre: «HB ist eine Filter-Zigarette, die schmeckt. $\mathrm{HB}$ rauchen heißt frohen Herzens genießen. » Dans le contexte des années 60, il n'y a rien de choquant à prêter au tabac des vertus apaisantes, et c'est cette loi de passage qui relie l'argument (vous êtes hors de vous) à la conclusion (fumez une cigarette $\mathrm{HB}$ ).

Le recours à ce "pastiche de genre» qu'est la publicité-dessin animé (cf. Bonhomme et Pahud 2013: 35) permet de désamorcer le caractère anxiogène des situations de crise construites de toutes pièces pour assurer la promotion du produit. Et puisque c'est par maladresse que le protagoniste se retrouve dans des situations périlleuses, le spectateur peut sans complexe rire des malheurs de Bruno.

\section{L'absence du produit appelle la crise (produit > crise)}

Parfois, ce n'est pas une crise préexistante qui rend nécessaire l'utilisation du produit ou du service (taches récalcitrantes > détachant; situation de stress $>$ cigarette), c'est la non-utilisation du produit qui provoque la crise, voire la catastrophe. Ces publicités présentent donc des exemples à ne pas suivre. On n'est plus ici dans une logique de victimisation du personnage publicitaire pouvant éventuellement susciter la compassion, mais plutôt dans une logique de culpabilisation du personnage, dont le consommateur est invité à se désolidariser: dans les années 90, une série de spots publicitaires humoristiques pour les Gelbe Seiten incitent les bricoleurs du dimanche à utiliser ce service pour s'épargner bien des déconvenues. Tantôt c'est le carrelage mural de la nouvelle salle de bains qui dégringole dans la baignoire où un couple s'est confortablement installé, une coupe de champagne à la main, pour fêter la fin des travaux:

Gelbe Seiten: Badewanne (1992) ${ }^{11}$

Tantôt c'est un mécanicien amateur qui met sa vie et celle de sa petite amie en danger après avoir bricolé lui-même sa voiture: ils sont à peine sortis du garage que la capote du cabriolet se rabat violemment sur leur tête:

11. Film publicitaire: [https://youtu.be/damCO6x_4NE]. 
Gelbe Seiten: Cabrio-Verdeck (1998) ${ }^{12}$

La morale de l'histoire est invariablement énoncée par la voix off en fin de spot: «Vielleicht hätte er / hätten sie jemanden fragen sollen, der sich mit so was auskennt. Gelbe Seiten machen das Leben leichter. » C'est le manquement à une règle de bon sens qui provoque la catastrophe, alors qu'elle aurait pu être évitée: ils ne se sont pas adressés à un professionnel (argument), donc ils en subissent les conséquences (conclusion).

Plus récemment, une campagne pour un fameux fromage français met en scène une jeune femme pas très futée ${ }^{13}$ dont le seul tort est d'oublier le précieux fromage dans diverses circonstances: une excursion en voiture, une séance d'escalade, ou même son propre mariage:

Le Cantal: baptême d'escalade (2009) ${ }^{14}$

Le Cantal: weekend (2009) ${ }^{15}$

Le Cantal: mariage (2009) ${ }^{16}$

À la question rituelle de son compagnon "Chantal, t'as pas oublié le Cantal? », la jeune femme ne sait que répondre. La sanction est immédiate et la voix off énonce la règle de passage permettant de faire le lien entre cet oubli et la réaction disproportionnée du jeune homme (il l'abandonne au bord de la route, la laisse chuter dans le vide ou refuse de l'épouser): «Oublier le Cantal, ça peut être fatal ».

On pourrait multiplier les exemples, car ce ressort de l'argumentation par la conséquence est un classique en publicité. Le scénario fait le plus souvent appel à un anti-modèle (personnage idiot, trop sûr de lui, mal informé), dont les actes ont invariablement des conséquences désastreuses, ce qui est censé produire un effet repoussoir (cf. Perelman et Olbrechts-Tyteca 1992: 493). II s'agit de mettre en garde le spectateur contre ces comportements à risque, pour mieux le ramener ensuite sur la voie de la raison, celle qui consiste à acheter le produit ou le service vanté par l'annonceur.

\section{Vive la crise! La publicité « crisophile »}

On a vu précédemment que la crise représentait une menace pour le système, pouvant aller jusqu'à entraîner sa destruction. Mais la crise comporte aussi des aspects positifs, à commencer par la remise en question de l'ordre établi: «La crise a [...] toujours un aspect d'éveil. Elle montre que ce qui allait de soi, ce qui semblait fonctionnel, efficace, comporte au moins des carences et des vices »

12. Film publicitaire: [https://youtu.be/FRuTBFrtZ6U].

13. Sur la dimension sexiste de cette publicité et la polémique qu'elle a suscitée, voir Schnitzer (2013: 415-417).

14. Film publicitaire: [https://youtu.be/TfXOHNMCjg0].

15. Film publicitaire: [https://youtu.be/MafU0xzNeSE].

16. Film publicitaire: [http://dai.ly/xal2je]. 
(Morin 1976: 159). Outre cette prise de conscience salutaire, la nécessité de trouver une issue à la crise stimule également la recherche de solutions et la prise de décisions. La crise porte donc également en elle la possibilité d'un renouveau: « [la crise] met en marche, ne serait-ce qu'un moment, ne serait-ce qu'à l'état naissant, tout ce qui peut apporter changement, transformation, évolution » (Morin 1976: 163).

Dans les publicités crisophiles, le produit provoque ou accompagne une crise salutaire qui bouleverse les conventions poussiéreuses, la bienséance hypocrite, brise le carcan des habitudes. Dans ce spot publicitaire des années 80 , la crise est latente et le produit joue un rôle de catalyseur:

Crunch : la fanfare (1983) ${ }^{17}$

La scène s'ouvre sur un concert de rue, le chef d'orchestre dirige tranquillement sa fanfare face à un public peu enthousiaste, lorsqu'une jeune femme croque vigoureusement dans une tablette de Crunch «le chocolat qui croustille », ce qui déclenche un véritable cataclysme, au grand émerveillement de la protagoniste. Dans une campagne pour le journal Bild dans les années 2000, ce sont les enfants qui déclenchent une crise en mettant leurs parents dans des situations très embarrassantes parce qu'ils disent innocemment des vérités qui dérangent:

Bild-Zeitung: Nichts ist härter als die Wahrheit (2005) ${ }^{18}$

L'une des saynètes se déroule dans la salle d'attente bien remplie d'un médecin de ville; une petite fille interroge sa mère à haute voix: "Mama, kriegt man bei jedem Urlaub die Syphilis? » Cette campagne fonctionne sur le principe de l'analogie: la parole des enfants est comme celle de Bild, vraie, directe, sans concession, quelles que soient les conséquences. Le quotidien familier des scandales fait de la crise le prix à payer pour la défense de la vérité: argument (les enfants / les journalistes de Bild disent la vérité), conclusion / conséquence (ils provoquent des crises), loi de passage (dire la vérité provoque des crises).

Dans l'exemple suivant, c'est la thématique du couple qui se sépare qui est mise à profit pour promouvoir l'achat de mobilier. La crise s'est déjà déclenchée et le produit contribue à la surmonter:

IKEA : Neuanfang $(2013)^{19}$

Folle de désespoir, la jeune femme met son appartement sens dessus dessous avant de le réaménager en un tournemain avec la bénédiction de la marque: "Verlieb dich neu in dein Zuhause», conseille la voix off. La crise existentielle débouche sur une renaissance symbolisée par un changement de décor (si facile

\footnotetext{
17. Film publicitaire: [http://www.ina.fr/video/PUB3502555040/crunch-tablette-de-chocolatfanfare-version-2-video.html].

18. Film publicitaire: [https://youtu.be/sb27jV3fRkA].

19. Film publicitaire: [https://youtu.be/mhbdUIADrJY].
} 
avec lkea). Le produit, pourtant sans rapport avec la crise, est à la fois le moyen et l'expression d'un nouveau départ.

\section{Le shockvertising: prévention, provocation gratuite ou engagement militant?}

Apparu dans les années 90, le concept de shockvertising ou publicité choc est un procédé publicitaire qui, comme son nom l'indique, consiste à choquer le spectateur pour capter son attention et ainsi renforcer la mémorisation. Les images ou les propos choquants peuvent avoir un rapport direct avec le message à faire passer, comme c'est le cas des campagnes institutionnelles; elles peuvent aussi, lorsqu'il s'agit de campagnes commerciales, n'avoir aucun rapport avec le produit.

\section{Les campagnes de prévention}

Les campagnes de publicité non commerciales, dédiées à la prévention, s'appuient généralement sur l'argumentation par l'anti-modèle et par la conséquence pour mettre le spectateur en garde contre la dangerosité de certains comportements tels que la violence routière (alcool au volant, téléphone au volant, excès de vitesse), ou les addictions (alcoolisme, tabagisme, drogue). On peut également classer dans cette catégorie les campagnes de lutte contre le racisme, l'homophobie, les violences conjugales, etc. Le ton est généralement grave, voire solennel. En matière de prévention routière, le message est immuable: les comportements à risque provoquent des accidents. Le moyen le plus sûr de frapper les esprits est la mise en scène de l'argumentation par la conséquence:

Sécurité routière: tous touchés $(2016)^{20}$

Verkehrssicherheit : runter vom Gas (2013) ${ }^{21}$

La catastrophe (accident mortel) est montrée à l'image, la relation de cause à conséquence doit s'imprimer dans l'esprit du spectateur: il roulait trop vite ou elle n'a pas attaché sa ceinture, résultat: ils sont morts.

Dans l'exemple suivant, les concepteurs ont choisi l'humour noir pour frapper les esprits et sensibiliser les jeunes aux dangers de l'utilisation du téléphone portable sur la voie publique:

Anastase: le tour de magie $(2015)^{22}$

D'abord présentée comme un «tour de magie », la « disparition » annoncée d'un jeune homme signifie en réalité qu'il va être fauché par une voiture au moment

20. Film publicitaire: [https://youtu.be/dIMfYq6KUTY]

21. Film publicitaire: [https://youtu.be/kF4rFgY3EKE].

22. Film publicitaire: [https://youtu.be/k8c2hjiTDPo]. 
où il traverse la rue distraitement, les yeux rivés sur son portable; le principe est le même qu'en (14) et (15), mais l'effet de surprise provoqué par ce scénario macabre et le ton cynique du narrateur (qui se révèle finalement être entrepreneur de pompes funèbres) rendent le choc émotionnel encore plus violent.

Si l'on peut comprendre que les campagnes institutionnelles aient recours à de tels procédés pour susciter une prise de conscience salutaire chez le public cible, l'intérêt du shockvertising dans le cadre de publicités commerciales paraît moins évident. Certaines marques se sont pourtant spécialisées dans ce créneau. On citera ici Sixt et Benetton qui, dans des styles très différents, ont choisi de choquer pour améliorer la notoriété de la marque.

\section{Sixt et la publicité-provocation}

La marque de location de véhicules a fait de la provocation sa stratégie de communication. Ses publicités exploitent volontiers des affaires touchant des personnalités publiques ou des sujets d'actualité qui font débat, pour les tourner en dérision en prétendant que la résolution de la crise passe par la location d'un véhicule Sixt. En 2009, la ministre de la santé Ulla Schmidt suscite la polémique en Allemagne pour avoir utilisé sa voiture de fonction lors d'un séjour privé en Espagne. Sixt en fait une occasion de promouvoir ses services:

Sixt: der Dienstwagen (2009) ${ }^{23}$

En encourageant la ministre à louer un véhicule Sixt (« Mit dem Dienstwagen in Urlaub? Es gibt Sixt doch auch in Alicante! »), la marque récupère et amplifie le bruit médiatique suscité par cette affaire pour attirer l'attention du public. Une crise bien réelle joue ici le rôle d'accroche publicitaire. Une constante des publicités Sixt réside donc dans la récupération de situations de crise à des fins commerciales, mais sur un mode humoristique, le client étant censé comprendre le second degré. Mais la stratégie peut aussi se retourner contre la marque, comme l'illustre pour cette affiche diffusée en 2011, en pleine période de discussion sur une possible sortie de la Grèce de la zone euro:

Sixt: Griechenlandkrise (2011) ${ }^{24}$

Annoncer aux Grecs que la marque accepte à nouveau les drachmes («Liebe Griechen, Sixt akzeptiert wieder Drachmen!»), dans un contexte de tension politique entre l'Allemagne et la Grèce est un exercice risqué et les conséquences ne se font pas attendre: I'affiche fait scandale en Grèce comme en Allemagne, la presse alimente la polémique, le patron de Sixt reçoit des menaces de mort et doit présenter des excuses. Dans ce cas précis, ajouter la crise (diplomatique) à la crise (politique) se révèle finalement contre-productif.

23. Affiche publicitaire: [http://images.derstandard.at/t/12/2009/07/29/1246621195053.jpg].

24. Affiche publicitaire: [http://ideen.fairmittlung.biz/wp-content/uploads/2011/07/sixt_griechenland.jpg]. 


\section{Benetton et la publicité militante}

Comme certaines publicités Sixt, les campagnes Benetton ont souvent suscité la controverse, mais dans un registre totalement différent. Alors que chez Sixt, la récupération commerciale de la crise est parfaitement assumée, Benetton brouille davantage encore les codes de la communication publicitaire en revendiquant l'utilisation de ce média à des fins non commerciales. Dans les années 80, les campagnes "United Colors» sont déjà engagées contre le racisme et la xénophobie, mais la forme se veut consensuelle:

Benetton: Happy New Year (1987) ${ }^{25}$

Lesannées 90 constituent un tournant.Lesscènes joyeuses montrant despersonnes de toutes origines portant des vêtements multicolores font progressivement place à des images chocs sans lien avec les produits. Certaines affiches provoquent des vagues d'indignation dans le monde entier: un malade du sida agonisant sur un lit d'hôpital, une fesse tatouée "HIV Positive", les vêtements maculés de sang d'un soldat décédé, ou encore des portraits de prisonniers condamnés à mort:

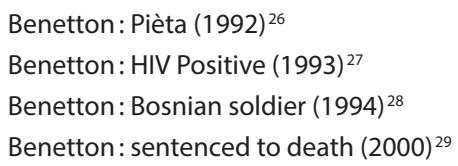

Luciano Benetton revendique un engagement sociétal et affirme que ses campagnes controversées ne sont pas motivées par des préoccupations marchandes, les produits ne sont d'ailleurs plus montrés à l'image. La publicité se trouve détournée de sa fonction commerciale pour devenir un acte militant. L'objectif n'est pas, selon Benetton, de vendre des pullovers, mais de dénoncer des injustices, de défendre des causes. II se justifie en affirmant que « ces photos sont autant de métaphores illustrant la discrimination pratiquée par la société à l'égard de ceux qui sont différents ${ }^{30} »$. Autrement dit, le message n'est pas au service de la publicité, mais c'est au contraire la publicité qui est mise au service du message. La crise n'est pas un prétexte pour faire vendre, elle devient un instrument au service d'un engagement militant. Dans une interview donnée au journal Der Spiegel en 1994, Luciano Benetton répond au journaliste l'accusant de cynisme que c'est du côté de la publicité traditionnelle qu'il faut chercher le

25. Film publicitaire: [http://www.ina.fr/video/PUB3784064169/benetton-vetement-hommefemme-enfant-video.html].

26. Affiche publicitaire: [https://abercrombiejames27.files.wordpress.com/2013/08/photoguides_ benetton_david_kirby_aids.jpg].

27. Affiche publicitaire: [http://img.e-marketing.fr/Images/Breves/Actus/48646/image/slide_251 312_1536490_huge.jpg].

28. Affiche publicitaire: [http://c0248141.cdn.cloudfiles.rackspacecloud.com/0000_00000_0021 464A.JPG].

29. Affiche publicitaire: [http://www.lexpress.fr/medias/1593/815694_benetton.jpg].

30. Citation reprise notamment dans Ardisson / Drouhet / Vebret 2010. 
cynisme: «Wir verbreiten keine Lügen, wir sagen, in dieser Welt gibt es Krankheit, Krieg und Tod » (Benetton 1994 [en ligne]). Appréciées des observateurs avertis, ces campagnes très clivantes ont pourtant détourné de la marque une partie de sa clientèle qui n'a jamais cru à la sincérité de cette démarche, et a considéré qu'il n'y avait là rien d'autre à voir que l'exploitation de la misère du monde à des fins commerciales ${ }^{31}$.

\section{Pour conclure}

L'objectif de cette étude était de montrer comment les campagnes publicitaires parviennent à concilier la mise en scène de crises ou de catastrophes avec l'éloge d'un produit, d'un service ou d'une marque en vue de faire acheter le produit au consommateur, ou de sensibiliser l'opinion à une cause, ou encore d'inciter les citoyens à changer de comportement. Si l'évocation de situations de crise, susceptible de provoquer des réactions négatives, peut sembler contreproductive au premier abord, elle remplit une fonction d'accroche indispensable en publicité. Le fait de frapper les esprits permet d'accroître les chances de mémorisation d'une campagne et donc d'augmenter la notoriété de la marque ou du produit vanté. Sur le plan argumentatif, la crise peut être présentée comme l'argument qui justifie l'utilisation du produit: ou bien le produit résout la crise, petite ou grande, et tout rentre dans l'ordre (Vanish, Vittel, HB), ou bien la crise est présentée comme la conséquence de la non-utilisation du produit, et il est alors trop tard pour l'endiguer, voire pour éviter la catastrophe (Gelbe Seiten, Le Cantal) et le spectateur est invité à se désolidariser de l'anti-modèle mis en scène dans la publicité. Les publicités chocs dédiées à la sécurité routière fonctionnent aussi sur ce principe. Dans le cas des publicités "crisophiles», deux variantes ont également été mises en évidence: ou bien le produit provoque une crise salutaire (Crunch, Bild), ou bien c'est la crise salutaire qui appelle l'utilisation du produit (Ikea). Quant aux campagnes commerciales relevant du shockvertising, elles s'appuient sur une longue tradition d'exploitation de la crise en publicité, tout en s'affranchissant des conventions du genre. L'originalité de Sixt est de tourner en dérision le principe de la Problemlösung en présentant artificiellement le produit comme une solution à une crise sans rapport avec celui-ci, alors que Benetton prétend rejeter toute logique commerciale en revendiquant l'absence totale de lien entre les produits de la marque et les crises montrées par les campagnes publicitaires qu'elle finance. L'argumentation se situe au niveau du métadiscours: montrer les crises que personne ne veut voir pour en dénoncer les causes (xénophobie, homophobie, toutes sortes de préjugés), c'est indirectement faire de l'engagement en faveur des droits humains un argument de vente.

31. Voir notamment l'article de l'Express du 25.08.1994 intitulé «Benetton a mal à sa pub », [http:// www.lexpress.fr/informations/benetton-a-mal-a-sa-pub_599227.html]. 


\section{Bibliographie}

Adam, Jean-Michel / Bonhomme, Marc (1997) L'Argumentation publicitaire: rhétorique de l'éloge et de la persuasion. Paris: Nathan.

Andersson, Bo (1997) «Ist ein “Muh!" ein relevantes Argument? Überlegungen zur Argumentation in der Werbung ». In: Bo Andersson / Gernot Müller (Hrsg.), Kleine Beiträge zur Germanistik. Festschrift für John Evert Härd, Uppsala: Acta Universitatis Upsaliensis, p. 17-32.

Ardisson, Thierry / Drouhet, Cyril / Vebret, Joseph (2010) Dictionnaire des provocateurs. Paris: Plon.

Benetton, Luciano (1994) «Krieg ist Realität ». Der Spiegel, 28.02.1994, [http:// www.spiegel.de/spiegel/print/d-13685044.html], dernière consultation le 30.10.2016.

Bonhomme, Marc / Pahud, Stéphanie (2013) «Un renouveau actuel de la rhétorique publicitaire?». Semen 36, p. 21-39.

Desîlets, Christian / Gauthier, Gilles (2013) «L'usage de l'argumentation en publicité ». Communication. Information médias théories pratiques 32 / 2, [https://communication.revues.org/4984], dernière consultation le 30.10.2016.

Gau, Daniela (2007) Erfolgreiche Werbung im interkulturellen Vergleich: eine Analyse deutsch- und französischsprachiger Werbung. Tübingen: Narr.

Morin, Edgar (1976) «Pour une crisologie ». Communications 25, p. 149-163.

Morin, Edgar / L'Yvonnet, François (2016) Pour une crisologie. Paris: L'Herne.

Perelman, Chaïm / Olbrechts-Tyteca, Lucie (1992) Traité de l'argumentation. La Nouvelle Rhétorique. Bruxelles: Éditions de I'Université de Bruxelles.

Schnitzer, Nathalie (2013) "“Chantal, t'as pas oublié Le Cantal?" Discours publicitaire et rôles sexués». In: Wolfgang Fink / Ingrid Haag / Katja Wimmer (Hrsg.), Frankreich-Deutschland: Transkulturelle Perspektiven France-Allemagne: Perpectives Transculturelles, Frankfurt a.M.: Peter Lang, p. 407-424.

Séguéla, Jacques (2004): « Notre métier est en danger et doit être défendu ». Les Échos, 01.07.2004, [http://www.lesechos.fr/01/07/2004/LesEchos/19191064-ECH_notre-metier-est-en-danger-et-doit-etre-defendu. htm\#wIS4dS6rGmKWQgTW.99], dernière consultation le 30.10.2016.

Thom, René (1976) «Crise et catastrophe ». Communications 25, p. 4-38.

Toulmin, Stephen (1993) Les Usages de l'argumentation. Trad. Ph. De Brabanter, Paris: Presses Universitaires de France. 